\title{
Efficient Segmentation of Medical MRI Image using WT-WS Algorithm
}

\author{
K.S.Tamilselvan \\ Assistant Professor (SGR), Dept of ECE, \\ Velalar College of Engineering and Technology, \\ Erode, TamilNadu, INDIA
}

\author{
G.Murugesan, PhD. \\ Professor and Head, Dept. of ECE, \\ Kongu Engineering College, Perundurai, \\ Tamilnadu, India
}

\begin{abstract}
Image segmentation is an important processing step in many image, video and computer vision applications especially in medical field. Extensive research has been done in creating many different approaches and algorithms for image segmentation, but it is still difficult to assess whether one algorithm produces more accurate segmentations than the other. Up to date, the most common method for evaluating the effectiveness of a segmentation method is subjective evaluation, in which a human visually compares the image segmentation results for separate segmentation algorithms, which is a tedious process. The use of computer-aided diagnosis (CAD) systems to improve the sensitivity and specificity of tumor detection has become a focus of medical imaging and diagnostic radiology research. Accurate segmentation of medical images is an important step in contouring during radiotherapy planning. This paper provides new hybrid medical image segmentation method based on Watershed and Wavelet Transform. In this paper, we propose an algorithm for segmentation problems in medical imaging modalities especially for brain Magnetic Resonance Images MRI). The watershed transformation is a useful morphological segmentation tool used for a variety of greyscale images. The segmentation procedure consists of pyramid representation, image segmentation, region merging and region projection. Each layer is split into a number of regions by rooting, labeling technique and the boundary is extracted by threshold and the image is smoothened by wavelet transform. Experimental results prove that the proposed algorithm is comparatively better than the existing systems .
\end{abstract}

\section{Keywords}

Computer-Aided Diagnosis, Magnetic Resonance Image, Contouring, Watershed Transformation, Wavelet Transform, Grey-scale Image, Pyramidal Image Segmentation.

\section{INTRODUCTION}

Image processing plays a vital role in diagnosis and analysis of various medical imaging modalities such as Xrays, PET, CT and MRI. Quantification by manual tracing of outlines of structures is tedious and time consuming. Image segmentation techniques results robust and high degree of accuracy in analyzing the image modalities [9]. Various categories of segmentation are clustering, edge detection and region extraction.
There are many image segmentation techniques available starting from region split and merge to morphological operation based segmentations [1] . The main disadvantage of the watershed based image segmentation algorithm is oversegmentation of the image due to the noise[3]. This drawback can be avoided by introducing a new image segmentation technique which uses both wavelet transform and watershed segmentation algorithm. This system uses Wavelet transform first which gives the low pass filtered image and this image is applied to the watershed technique to avoid the noise regions.

\section{SEGMENTATION METHOD}

Original MRI/CT image is applied to the discrete wavelet Transform to decompose the image which allows a perfect reproduction of original image, so that it is called as a complete representation. Wavelet Transform is a mathematical tool that can be used to describe the images in multiple resolutions [12]. As a low pass filter is involved, it is easy to implement noise suppression in this method.(Images are first segmented into a number of regions at each layer of the pyramid with watershed transformation[3]. Starting from top layer, regions are merged with merging parameter. The resulting image is projected onto the next layer by inverse wavelet transform until the wavelet decomposition level $\mathrm{L}$ equals 0).

\subsection{Morphological filtering method}

The object to be segmented differs greatly in contrast from the background image[13].For obtaining the proper segmentation of an image especially for medical images, the segmentation algorithm need to be contain the following step compulsorily. Preprocessing of the image which includes smoothing of the image and removal of noise in the image[13]. As these factors are affecting the segmentation efficiency, these steps are important in image segmentation.

All the medical images are containing both smoothing and detailed information. The smoothing information can be easily filtered out by using more number of morphological filters [1]. Because of this The very small-scale details are usually considered. Therefore by using the above technique smooth gray- level images can be obtained with a composition of opening and closing of a given structuring element. The main drawback of normally used opening and closing is that it does not permit a perfect preservation of the edge information and their shape completely. In this paper, the wavelet transform used is preserve the edge information of the tumor 
from the MRI image of a human brain also by removing the noise areas of the image.

Noise in the acquired MRI image is suppressed by smoothening the image based on the thresholding technique[5] of Discrete Wavelet Transform and then reconstructing the original image by taking the inverse of Wavelet Transform. The most of the noise tends to be represented by wavelet coefficients at finer scale. By omitting the wavelet coefficients, we will obtain the morphological filtering of the noise in the image. This can be done by making the wavelet coefficient equal to zero, if the value is less than a threshold value which is calculated by standard deviation of the sub bands. As per the proposed algorithm, denoised and smoothed image can be obtained by taking inverse wavelet transform of the co-efficient.

\subsection{Pyramidal representation}

Multiresolution techniques are used to obtain the complete view and analysis of an image by examining it at various resolution levels creating the pyramidal representation of the image[10]. The available types include Gaussian Pyramids, laplacian Pyramids and wavelets. The first two methods experience some information loss where as the third method provides a maximum level of decomposition. Wavelets are functions generated from a single function by its dilations and translations. The Haar transform is one of the best suitable method to create a multiresolution image[6].

The Haar wavelet transform of a signal $\mathrm{f}(\mathrm{x})$ is calculated by convolution of the signal $\mathrm{f}(\mathrm{x})$ with basis function as,

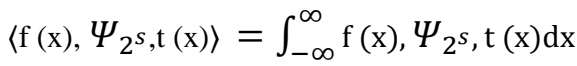

Where the second term within the integral $\Psi 2(\mathrm{x})$ mentions the basis function. The variables ' $\mathrm{s}$ ' and ' $\mathrm{t}$ ' are dilation and translation parameters respectively. Here we can also use the Mallat's pyramid algorithm [12] to find the wavelet coefficients to decompose the given medical image into its constitute parts.

Steps to be followed when using wavelet decomposition of an image:

1.Seperate the given image into LL.LH.HL and $\mathrm{HH}$ using low pass filter at a scale of $2 \mathrm{j}$

2.Repeate the process of decomposition on LL sub band until the no. of sub bands are equal to 4 .

3. Find the $\mathbf{J}$ scale transforms, for the original image as represented by

$$
\begin{array}{r}
\left(W^{2^{i}},\left(W_{H}^{2^{i}} \quad W_{V}^{2^{i}} W_{D}^{2^{i}}\right) 1 \leq i \leq J\right) \ldots \ldots \ldots \ldots \\
\text { for } j=1,2, \ldots, J
\end{array}
$$

where the size of the wavelet representation is same as original signal.

\subsection{Image Segmentation and \\ Segmentation Projection}

The watershed transformation method considered as a difficult segmentation operation comparing with other image segmentation techniques. This method is useful in medical field because it is solve the overlapping between the closest grey levels in medical images [11].

Watershed segmentation technique is applied to the images with different levels of resolutions, after forming the pyramidal image using wavelet transform. Normally the noise affected images in each layer of this pyramid is implemented for segmentation.

\subsection{Steps followed in applying Watershed Algorithm:}

STEP 1: Read the Original image (I0).

STEP 2: Take L level wavelet transform of Image (IL)

STEP3: Image smoothed and the noise particles are removed by wavelet De noising Technique

STEP 4: Apply watershed segmentation

STEP 5: Resulting merged Region image is Projected onto the $\mathrm{I} L$ - 1 layer by an Inverse wavelet transform

STEP 6: Go to step3 and repeat the above procedure until $\mathrm{L}$ equals 0

Read Image:

Read the given MRI image, which is the medical image got from MRI Scanner.

\section{Maximize Contrast:}

To minimize the number of valleys found by the watershed transform, maximize the contrast of the objects of interest. Common techniques for contrast enhancement of an image, by wavelet transform. An original image is decomposed into ' $\mathrm{n}$ ' levels by Daubechies4 (db4) wavelet transform (2D-DWT).

In this method first compute $\alpha_{n, s}$ by using the following equation .

Let ' $n$ ' is level,

$\mathrm{E}_{\mathrm{n}, \mathrm{s}}=\frac{\sum_{i, j}\left|x_{n, s}(i, j)\right|}{N}$

$\alpha_{n, s}=\frac{E_{n+1, s}}{\sum_{S} E_{n+1, S / 2}}$

where's' is the sub band which indicates LH, HL and HH. $\mathrm{E}_{\mathrm{n}, \mathrm{s}}$ is the average absolute value of a specific sub band $s$ in level $n . \mathrm{N}$ is number of coefficients of each sub band.

$\bar{x} \mathrm{n}, \mathrm{s}(\mathrm{i}, \mathrm{j})$ is enhanced sub band from each sub band $x_{\mathrm{n}, \mathrm{s}}(\mathrm{i}, \mathrm{j})$ computed by Eq. 2.3 .

$\bar{x} \mathrm{n}, \mathrm{s}(\mathrm{i}, \mathrm{j})=\alpha_{n, s} x_{n, s}(i, j)$ 
Compute the enhanced image for each sub band and reconstruct the image using inverse wavelet transform. Resulting image is the enhanced image.

\section{Convert Objects of Interest:}

Watershed transform detects intensity "valleys" in the image. The enhanced image is converted into the objects of interest with high intensity valleys [2].

\section{Detect Intensity Valleys:}

All the intensity valleys are below a particular threshold .The output of the extended min function is a binary image. The location rather than the size of the regions is important. The min function modifies the image to contain only those valleys found by the extended min function. The min function will also change a valley's pixel values to zero (deepest possible valley for uint8 images). All regions containing an imposed minimum will be detected by the watershed transform.

\section{Watershed Segmentation:}

Watershed segmentation of the imposed minima image is accomplished with the watershed function. The watershed function returns a label matrix containing nonnegative numbers that correspond to watershed regions. Pixels that do not fall into any watershed region are given a pixel value of zero.

\section{Merging over-segmentation regions:}

The watershed transforms is sensitive to the change of the data therefore the noise objective brim and thin changes inside objectives can lead to over-segmentation, over segmentation is got by the method from watershed transform, the same aim or the same background is divided into several parts. The conditions for combining rules are:

a) The regions that will be combined are neighbors.

b) The characteristics of the region, which will

be combined should be similar.

c) The big region after combining is useful.

To merge the region which are neighbors and similar can be according to the similitude. If similitude is higher than threshold, then the two neighbor fields can be seen as the same region and that can be combined. After combination, the background and the aim are separated. Usually, similitude can be defined according to gray, texture, and so on.

Suppose $\mathrm{Bi}$ is set of boundary points of catchment basin I ( $\mathrm{CBi})$, Ni stands for the number of $\mathrm{Bi}$, then the boundary gray mean of the $\mathrm{CBi}$, The definition of $\mathrm{B}$ mean (i) is given by

$$
B \text { mean }(i)=f(p j) / N i
$$

Normally, the gray mean of the whole CB is applied as the standard for joining neighbor areas, but because the main dissimilarity among each $\mathrm{CB}$ of medical images after the watershed transforms lies in boundary. The method is to calculate gray mean of boundary points. It not only reduces the work of calculation but also neglects the side factors which affect judgment and decision; it also stresses the primary factors.

In medical image due to the effect of noise, many small $\mathrm{CB}$ are produced and these small $\mathrm{CB}$ are combined to form the large image[3].

Once the merged image $\mathrm{M}$ is generated at $\mathrm{L}^{\text {th }}$ layer and it must be projected onto next layer in order to finish the full resolution image segmentation. Direct projection of the segmented image offers very poor results. To overcome the problem inverse wavelet transform is used to implemented from low to high resolution layer step by step.

During projection from $\mathrm{I}^{\text {th }}$ layer to I-1th layer, a parentchild spatial relationship between the image elements of two successive layers is defined. This relationship is evaluated by means of a similarity measure. The children of a layer can belong to different parents in the upper layer. Similarity between a child image element and its possible parents describes the similarities. By feature of image elements similarity is defined, for example by comparing the contrast or texture properties of a child and its possible parents.

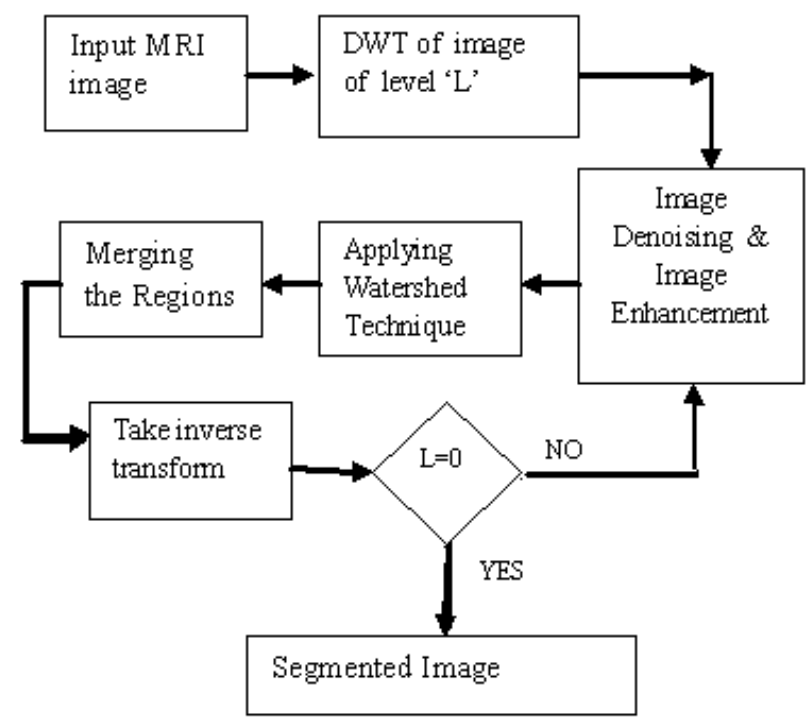

Figure 2.1: Block diagram representation of Proposed Image Segmentation Technique

\section{FEATURE EXTRACTION \& QUALITY EVALUATION}

We use the characters Root Mean Square Error (RMSE), Peak Signal to Noise Ratio (PSNR) and Average Difference (AD) of the resultant images of different techniques used in this paper to compare performance analysis of the existing methods with a new method . From the results it is clear that the proposed method gives a better performance than the others.

\section{Root Mean Square Error:}

RMSE is used to measure the difference between the source image and the segmented image, the smaller the value of RMSE and the smaller the difference, the better the fusion performance.

RMSE $=\sqrt{\frac{\sum_{i=1}^{M} \sum_{\mathrm{j}=1}^{\mathrm{N}}[\mathrm{M}(\mathrm{i}, \mathrm{j})-\mathrm{F}(\mathrm{i}, \mathrm{j})]}{\mathrm{MXN}}}$

Where $\mathrm{M}$ and $\mathrm{N}$ are row and column, $\mathrm{I}$ and $\mathrm{j}$ are the elements in the row and column.

Peak-to-peak Signal to Noise Ratio:

The bigger the value of PSNR, the better the segmentation performance.

$P S N R=10 \square \ln \left(f_{\max } / R M S E\right) 2$ 


\section{Average Difference $(A D)$ :}

Average Difference is the difference in each pixel value between the input image and the segmented image. The lower the value of $\mathrm{AD}$, the better the segmentation performance.

\section{RESULTS AND DISCUSSION}

Initially the MRI image is read from a MRI scanner and this is shown in figure $4.1 \& 4.8$. Enhanced image is as shown in fig 4.2.\& 4.9. The aim of this project is to extract the boundary of tumor area in brain image. The input brain MRI image is then subjected to second level decomposition using wavelet transformation. This is shown in figure $4.3 \& 4.10$.

One level decomposition Produces HH, HL, LH, LL images .On two level Decomposition HHHH, HHHL, LLHL, LLLL is got. This LL image on watershed transform overcomes over segmentation. Boundary extraction of LL image using watershed algorithm is shown in the fig $4.4 \&$ 4.11.Boundary superimposed on first level decomposed image (LL) is shown in the fig $4.5 \& 4.12$. Boundary extraction of synthesized image by watershed algorithm is shown in the fig $4.6 \& 4.13$. The boundary is superimposed on the original image as shown in figure $4.7 \& 4.14$.

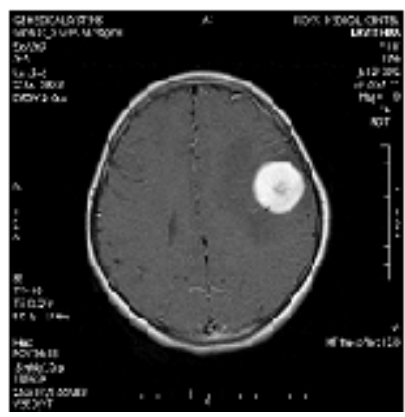

Fig 4.1: Original MRI Brain Image

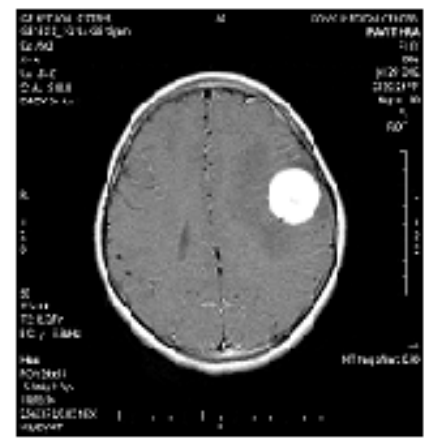

Fig 4.2: Enhanced Image

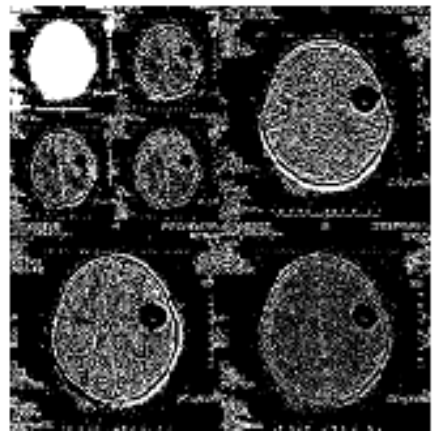

Fig 4.3: Two level Decomposition of enhanced image

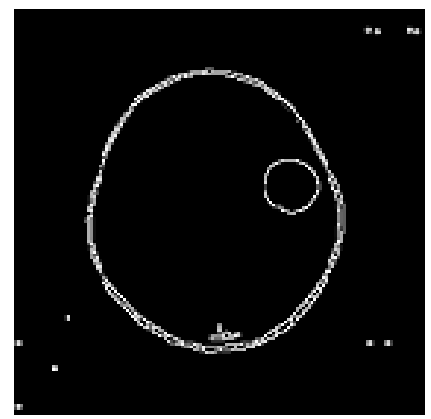

Fig 4.4 Boundary extraction of $L L$ image using watershed algorithm

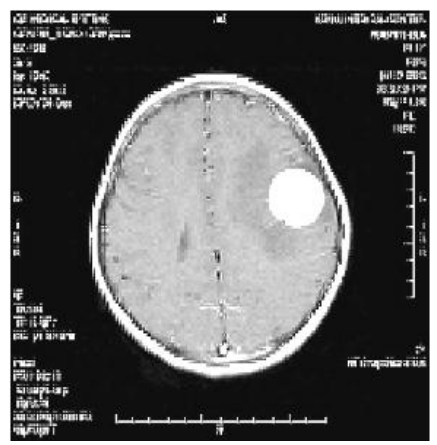

Fig 4.5: Boundary superimposed on first level decomposed image(LL)

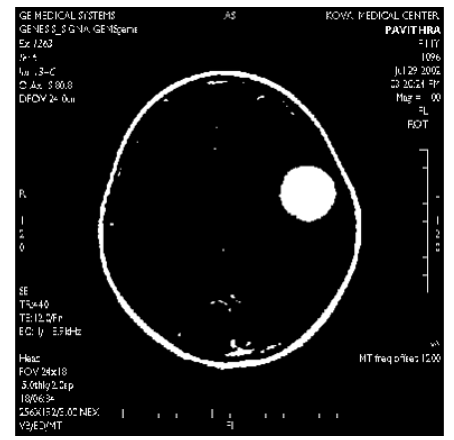

Fig 4.6: Boundary extraction of reconstructed image using watershed algorithm 


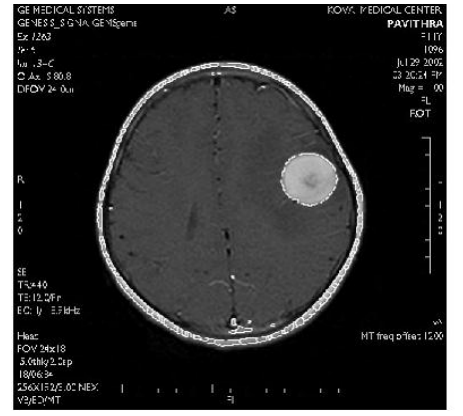

Fig 4.7: Boundary superimposed on original image

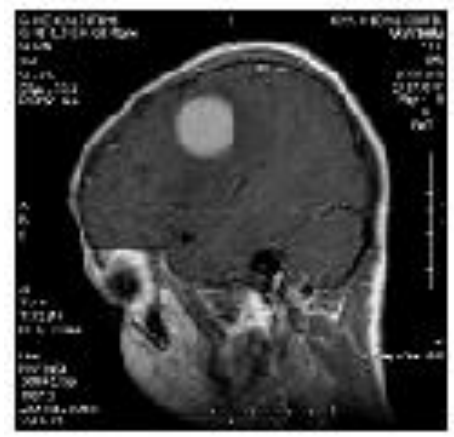

Fig 4.8: Original MRI image

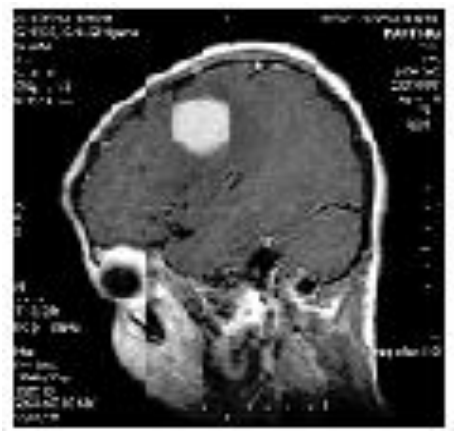

Fig 4.9: Enhanced Image

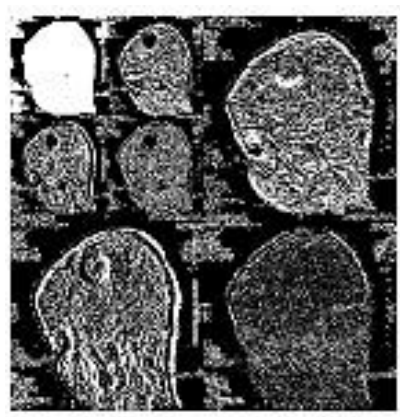

Fig 4.10: Two level Decomposition of enhanced image

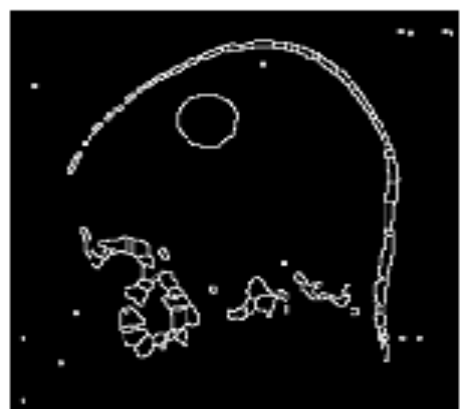

Fig 4.11: Boundary extraction of LL image using watershed algorithm

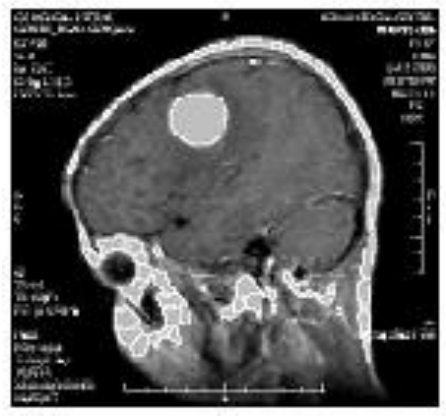

Fig 4.12: Boundary superimposed on first level decomposed image(LL)

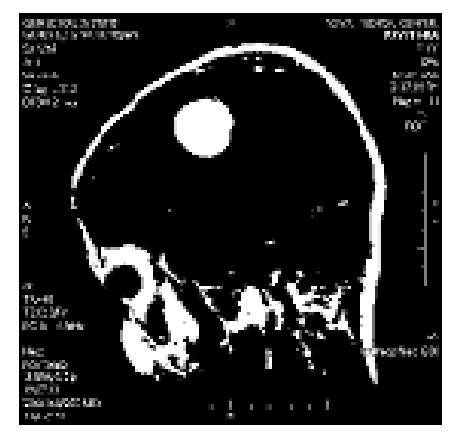

Fig 4.13: Boundary extraction of reconstructed image using watershed algorithm

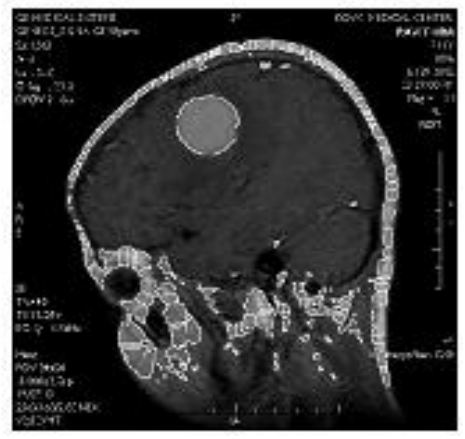

Fig 4.14: Boundary superimposed on original image 


\begin{tabular}{|c|c|c|c|}
\hline Method & RMSE & PSNR & AD \\
\hline Watershed & $\mathbf{0 . 0 3 1 2}$ & 55416 & 9.725e-004 \\
\hline Wavelet & $\mathbf{0 . 0 2 3 6}$ & 55434 & $5.714 \mathrm{e}-004$ \\
\hline $\begin{array}{c}\text { Proposed } \\
\text { Method }\end{array}$ & $\mathbf{0 . 0 1 5 4}$ & $\mathbf{5 5 4 9 8}$ & $\mathbf{2 . 3 6 1 1 e - 0 0 4}$ \\
& & & \\
\hline
\end{tabular}

Table-4.1: Comparison of RMSE, PSNR and AD

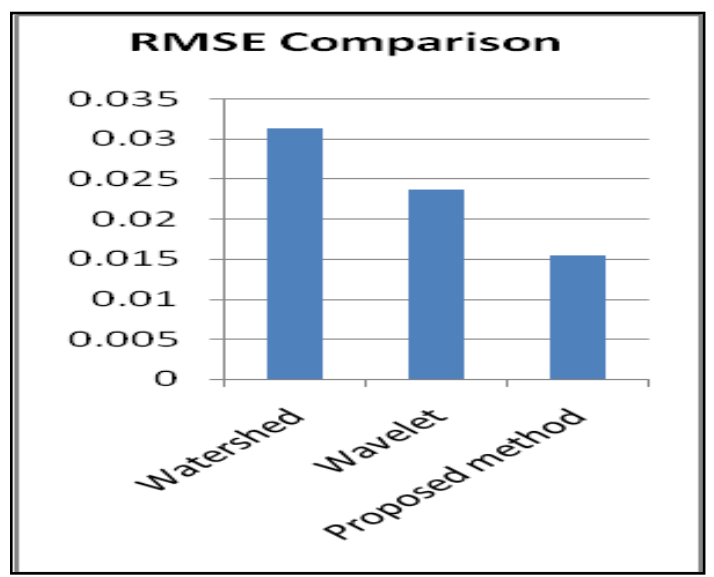

Fig.4.15.Comparison of measured values of RMSE for different Image Segmentation Techniques

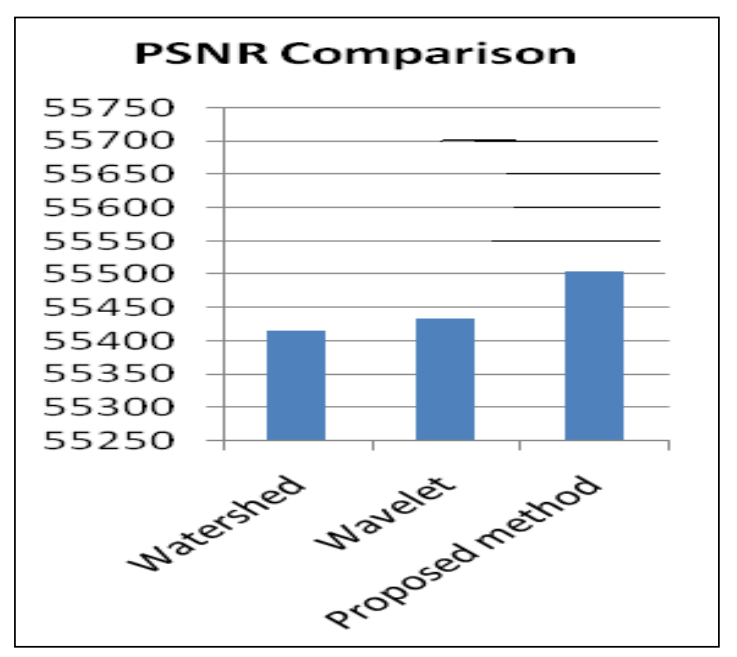

Fig.4.16.Comparison of measured values of PSNR for different Image Segmentation Techniques

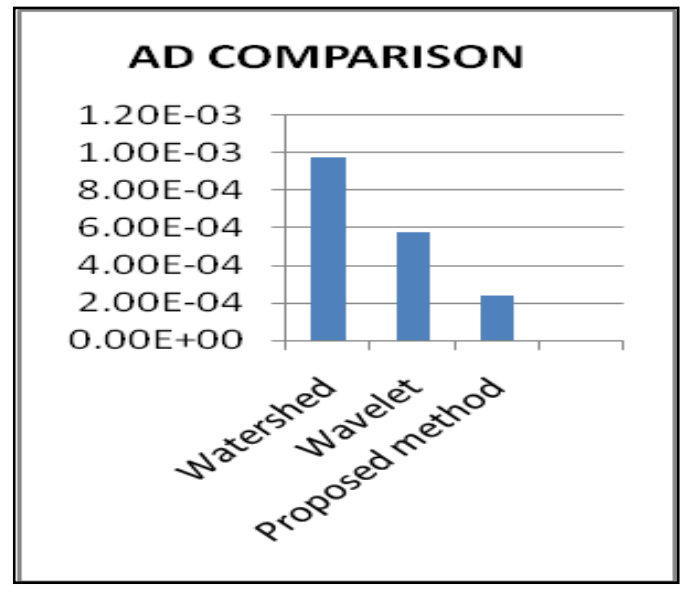

Fig.4.17.Comparison of measured values of $\mathrm{AD}$ for different Image Segmentation Techniques

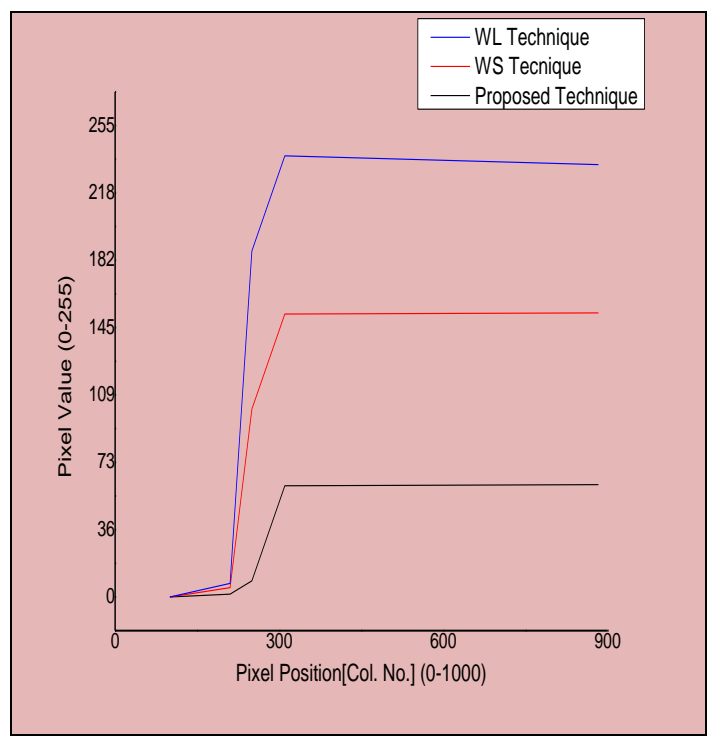

Fig.4.18.Comparison of measured values for different Image Segmentation Techniques

\section{CONCLUSION}

This paper proposes a method to detect the tumor in human brain. Earlier method of tumor extraction is concentrated on wavelet transform (or) watershed transform. But those methods had a drawback of over-segmentation due to noise. In this paper, we proposed a new multiresolution technique for image segmentation based on wavelet decompositions and watersheds. It allows resolution selection for applying the watershed transform, and projects the segmentation result back into the original full resolution, without loss of edge definition. The initial resolution must be chosen in accordance with the size and number of the desired objects. More and smaller objects are segmented when finer initial resolutions are selected, while less and larger objects are obtained for coarser resolution. The experimental results indicate that the proposed technique performs well for both inherent and artificial noise contamination. We have described how regions are projected onto lower layer and build hierarchical parentchild regions relationship of successive two layers. As shown 
in our experimental results, the algorithm generates visually meaningful segmentation results.

\section{ACKNOWLEDGMENTS}

The authors would like to thank Dr.A.Murugesan, Radiologist \& Dr.R.VinothSaravanan, Johnsons MRI Scan and Diagnostic Centre, Erode for providing us with sufficient technical assistance and valuable advice.

\section{REFERENCES}

[1] Chi-Man Pun and Hong-Min Zhu, "Image Segmentation Using Discrete Cosine Texture Feature" International Journal of Computers Issue 1, Volume 4, 2010

[2] Cla'udio Rosito Junga, Jacob Scharcanskib, "Robust Watershed Segmentation using Wavelets", Image and Vision Computing 23 (2005) 661-669

[3] Cla'udio Rosito Jung Unisinos, "Multiscale Image Segmentation Using Wavelets and watersheds" IEEE Trans, Image Process, 2003

[4] Gonzalez, R., C., Woods, R., E., "Digital Image Processing", 2nd ed., Prentice Hall, New Jersey, USA, 2002, chap 6, 7.

[5] Haihua Liu, Zhouhui Chen, Xinhao Chen, Yaguang Chen, "Multiresolution Medical Image Segmentation Based on Wavelet Transform", Proceedings of the 2005 IEEE Engineering in Medicine and Biology 27th Annual Conference, Shanghai, China, September 1-4, 2005

[6] Kasmir Raja.S.V, Shaik Abdul Khadir.A, Riaz Ahamed.S.S, "Moving Toward Region-Based Image Segmentation Techniques: A study" Journal of Theoretical and Applied Information Technology, 2009.
[7] Kother Mohideen.S, "Image Segmentation Based on Inhomogeneity Pixel using Complex Wavelet Transform for Medical Image", International Journal of Advanced Research in Computer Science and Software Engineering, Volume 2, Issue 5, May 2012

[8] Minal M. Purani, Prof, Shobha Krishnan, "Segmentation of Image Using Watershed and Fast Level set method Special Issue of IJCCT, Vol. 2 Issue 2, 3, 4; 2010 for International Conference [ICCT-2010], 3rd-5th December 2010.

[9] Preeti Aggarwal UIET,Renu Vig Sonali Bhadoria, C.G.Dethe,"Role of Segmentation in Medical Imaging: A Comparative Study", International Journal of Computer Applications, Volume 29- No.1, September 2011

[10] Rezaee,M.R., $\quad$ P.M.J.Lelieveldt,B.P.E，Vanergeest. , R.J.Reibert,J.H.C, “A Multiresolution in Segmentation Technique based on Pyramidal Segmentation and Fuzzy Clustering", IEEE Trans, Image Process, 2002.

[11] Roberto Rodriguez, Rubén Hernandez, "An Unsupervised Strategy for Biomedical Image Segmentation," Journal of Advances and Applications in Bioinformatics and Chemistry, 2010

[12] Stephane Mallat, "A Wavelet Tour of Signal Processing", Third Edition: The Sparse Way, Academic Press, 3rd edition, 2008.

[13] Saif D. Salman \& Ahmed A. Bahrani, "Segmentation of tumor tissue in gray medical images using watershed transformation method", International Journal of Advancements in Computing Technology, Volume 2, Number 4, October 2010 